\title{
Radial Distortion Self-Calibration
}

\author{
José Henrique Brito \\ IPCA, Barcelos, Portugal \\ Centro Algoritmi, Universidade do Minho, Portugal \\ josehbrito@gmail.com \\ Kevin Köser \\ ETH Zürich, Switzerland \\ kevin.koesereinf.ethz.ch
}

\begin{abstract}
In cameras with radial distortion, straight lines in space are in general mapped to curves in the image. Although epipolar geometry also gets distorted, there is a set of special epipolar lines that remain straight, namely those that go through the distortion center. By finding these straight epipolar lines in camera pairs we can obtain constraints on the distortion center( $s$ ) without any calibration object or plumbline assumptions in the scene. Although this holds for all radial distortion models we conceptually prove this idea using the division distortion model and the radial fundamental matrix which allow for a very simple closed form solution of the distortion center from two views (same distortion) or three views (different distortions). The non-iterative nature of our approach makes it immune to local minima and allows finding the distortion center also for cropped images or those where no good prior exists. Besides this, we give comprehensive relations between different undistortion models and discuss advantages and drawbacks.
\end{abstract}

\section{Introduction}

In the last two decades there has been tremendous progress in automatically reconstructing scenes and camera trajectories from images. In particular the theories for autocalibration allowed to exploit uncalibrated images, making it possible to register pictures from archives or photo collections where the physical camera hardware is unknown and unaccessible and thus no pre-calibration of imaging parameters is possible (cf. to [15] for an overview). However, these techniques usually assume ideal pinhole cameras and do not consider lens distortion. To compensate for such distortion present in many real lens systems,

${ }^{*}$ This work was done while this author was employed by the Department of Computer Science, ETH Zürich

\author{
Roland Angst* \\ Stanford University \\ ETH Zürich, Switzerland \\ rangstestanford.edu
}

\author{
Marc Pollefeys \\ ETH Zürich, Switzerland \\ marc.pollefeyseinf.ethz.ch
}

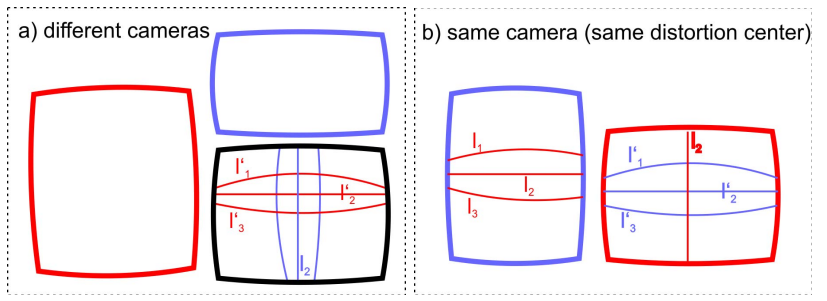

Figure 1. Epipolar curves $\left(l_{i}\right.$ and $\left.l_{i}^{\prime}\right)$ are (degenerated) circles in general, but those that go through the center of radial distortion are straight lines $\left(l_{2}\right.$ and $\left.l_{2}^{\prime}\right)$. a) When the radial fundamental matrix is obtained with respect to two other images (potentially with different or no distortion) the intersection of the straight epipolar lines reveals the CoD. b) In case the same camera is used to take a pair of images the $\mathrm{CoD}$ has to lie on the straight epipolar curves in both images.

most importantly radial distortion, several pre-calibration techniques based on calibration objects have been proposed $[4,24,16,14,25,22]$ that allow rectification of distorted images prior to further processing. This is however only an option in case the camera (and a calibration object) are accessible. Only few work has addressed autocalibration of radial distortion and virtually all of this focuses on the distortion strength and assumes the distortion center being known.

In this contribution we drop this assumption and show how to estimate the center of distortion $(C o D)$ from image data of completely unknown scenes without a calibration object, given a perspective (single center of projection) camera with strictly radial distortion. When projecting the viewing ray of another camera to some point in space into the view of such a camera, one does not obtain an epipolar line, but more generally an epipolar curve. However - under any radial distortion model - the epipolar curve that contains the $\mathrm{CoD}$ must be a straight line, since the distortion happens 
in direction of the line $e^{1}$. Consequently, for a pair of images we can simply search the set of all possible epipolar curves in the second image and pick the one that is straight, the CoD must lie on that line. Given a second straight epipolar curve from a different epipolar geometry (e.g. from a third camera) the $\mathrm{CoD}$ is uniquely defined (see also Fig. 1).

While these considerations apply to all radial distortion models, we chose to demonstrate the auto-calibration technique using the division distortion model proposed by Fitzgibbon [10], however using the lifted formulation by Brito et al. [3] where the $\mathrm{CoD}$ need not be known beforehand. The main reason for this is that the set of all epipolar curves is just the row space of the radial fundamental matrix and the search for the straight line becomes a linear problem. This allows us to obtain the solution in closed form, rather than performing a local optimization as performed e.g. in calibration toolboxes. This is in particular interesting for cropped images or those with digital zoom, where the CoD may even lie outside the image and no good prior guess is available. We also relate the model of Fitzgibbon [10], Barreto et al. [2] and Brito et al. [3] to the rational function distortion by Claus et al. [8] and point out differences, advantages and drawbacks.

\section{Previous Work}

Lens distortion has been studied in photogrammetry and computer vision for a long time (cf. to $[5,6]$ for a historical overview) and many procedures exist that can calibrate the intrinsic parameters of a camera given some calibration object $[4,24,16,14,25,22]$. Here, radial distortion is one of the dominant distortions [24] and can severely hurt image registration, reconstruction or measurement in images when not being considered. This is even more so since the advent of digital consumer cameras and cheap lenses that were not intended to almost perfectly obey a photogrammetric imaging model but merely to produce nice pictures [6].

On the other hand, fully automatic systems have been developed that can register video sequences (e.g. [20]), archive imagery or online photo collections (e.g. [21]), where the physical camera is no longer accessible or unknown. For cameras that strictly obey the perspective pinhole camera model, the intrinsic parameters can be obtained by means of self-calibration allowing again for metric measurements. However, given significant distortion most of these methods fail or require pre-rectification with known distortion parameters.

Only few authors have considered to include distortion into the self-calibration problem [10, 19, 2, 23, 8, 13, 3]. Among these, Thirtala and Pollefeys [23] assume the CoD to be known and reason about the shape of the distortion in

\footnotetext{
${ }^{1}$ Any line through the $\mathrm{CoD}$ is a fixed line when radially distorting or undistorting an image, although not a line of fixed points.
}

radial direction. Also Fitzgibbon [10] assumed the CoD to be known and then introduced an undistortion model rather than a distortion model allowing to work directly with distorted coordinates. This led to the $4 \times 4$ radial fundamental matrix with known CoD by Barreto et al. [2] that has been generalized to absorb an unknown CoD by Brito et al. [3].

After the division undistortion model of [10] also Claus and Fitzgibbon [8] generalize the model, now to a rational function undistortion where up to 17 distortion parameters exist, allowing to represent more general distortion functions, not just radial distortion. They also propose a local optimization method, that - given a good initial value - can obtain most of the parameters from images alone, although some ambiguity remains. To remedy this, in a later paper [7] they suggest to mark curves in the image that need to be straight when undistorted, which in practice means exploiting a known calibration object (the lines). This is an idea that also Devernay and Faugeras had followed [9]. The relations and differences of the models used in [10, 8, 7, 2, 3] will be discussed in more detail later.

Micusik and Pajdla [19] use the center of the viewing ellipse in an omnidirectional camera to start and then linearize the problem. This is not possible for (almost) perspective images, since there is no visible image border. Geyer and Daniilidis [11] formulated epipolar geometry for catadioptric views and estimated the principal point (the CoD) using the notion that such systems project lines into circles. Assuming a known $\mathrm{CoD}$, Barreto [1] later proposed a unifying model that covers catadioptric and perspective cameras with or without radial (barrel) distortion. Using a similar lifting framework as in [1], our work extends Geyer and Daniilidis' work [11] from catadioptric to perspective cameras with radial (barrel or pincushion) distortion.

$\mathrm{Li}$ and Hartley [18] point out it is important to estimate the $\mathrm{CoD}$ accurately rather than using the image center. They use the method of Hartley and Kang [13] to estimate it. Essentially, their idea is that when observing a planar calibration pattern with a camera with radial distortion, then the CoD will act like a focus of expansion or contraction and consequently behave similarly as an epipole. Consequently, it can be estimated by using a $3 \times 3$ fundamental matrix between the planar pattern and the distorted image. For the most general case that we also consider in this paper (unknown, non-planar scenes), they argue that still each corresponding point in the distorted images must lie on a line towards the $\mathrm{CoD}$. By using an 80-point algorithm in four views they argue it would be possible to construct the quadrifocal tensor, extract the cameras and then the CoDs. While from a geometrical point of view this is a very nice idea, in practice it would be very difficult and expensive to find a non-degenerate, outlier-free set of 80 correspondences across four views. Nevertheless, since this method is the only that could find an arbitrary radial CoD without ini- 
tialization and without calibration object, we consider this as the closest work to ours in the literature.

Our solution is based on the observation that lines that go through the $\mathrm{CoD}$ are fixed lines under distortion and undistortion. Consequently, we look for straight lines in the set of all possible epipolar curves and argue that the CoD must be on such a line. In order to obtain a parametric representation of the set of all possible epipolar curves, we use the generalization of Fitzgibbon's division distortion model by Brito et al. [3], where all epipolar lines are representable by quadratic curves. To the best of our knowledge, this is the first work that can extract the $\mathrm{CoD}$ from a pair (with same distortion) or triplet of unknown images (with different or no distortion). On top, we also estimate the distortion strength, which - in sum - is why we call this method radial distortion self-calibration.

\section{Relation between Undistortion Models}

The traditionally used second-order distortion model in computer vision (motivated in [4]) with $\mathrm{CoD}\left(d_{x}, d_{y}\right)^{T} \in$ $\mathbb{R}^{2}$ describes the radial distortion as

$$
\left(\begin{array}{l}
x_{d} \\
y_{d}
\end{array}\right)=\left(\begin{array}{l}
x_{u} \\
y_{u}
\end{array}\right)+\lambda \tilde{r}^{2}\left(\left(\begin{array}{l}
x_{u} \\
y_{u}
\end{array}\right)-\left(\begin{array}{l}
d_{x} \\
d_{y}
\end{array}\right)\right)
$$

where $\left(x_{d}, y_{d}\right)^{T} \in \mathbb{R}^{2}$ and $\left(x_{u}, y_{u}\right)^{T} \in \mathbb{R}^{2}$ are the distorted and the undistorted point, respectively, whereas $\lambda \in \mathbb{R}$ is the distortion coefficient and $\tilde{r}^{2}=\left\|\left(x_{u}, y_{u}\right)^{T}-\left(d_{x}, d_{y}\right)^{T}\right\|^{2}$ is the squared Euclidean distance between the $\mathrm{CoD}$ and the undistorted point. Eq. (1) is a distortion model since it actually describes the distorted point in explicit form: given the undistorted point $\left(x_{u}, y_{u}\right)^{T}$ and the distortion parameters $\lambda$ and $\left(d_{x}, d_{y}\right)^{T}$, the distorted point can be computed easily by evaluating the right-hand side of Eq. (1). For auto-calibration, or for direct estimation based on distorted measurements, an undistortion model rather than a distortion model is required. We will now compare different such models starting with the one proposed by Fitzgibbon [10].

The division undistortion model In [10], Fitzgibbon argues that an undistortion model can be equally powerful as a distortion model and compares several (un-)distortion functions. The basic equation expresses the undistorted point with homogeneous coordinates as

$$
p_{u}=\left(\begin{array}{c}
x_{u} \\
y_{u} \\
1
\end{array}\right) \cong\left(\begin{array}{c}
x_{d} \\
y_{d} \\
1+\lambda r^{2}
\end{array}\right)
$$

with $r^{2}=x_{d}^{2}+y_{d}^{2}$ and where $\cong$ denotes equality up to a scalar multiple. This is the model also used in the radial fundamental matrix with lifted coordinates [2] and later by minimal solvers with radial distortion like [17]. Since $r^{2}$

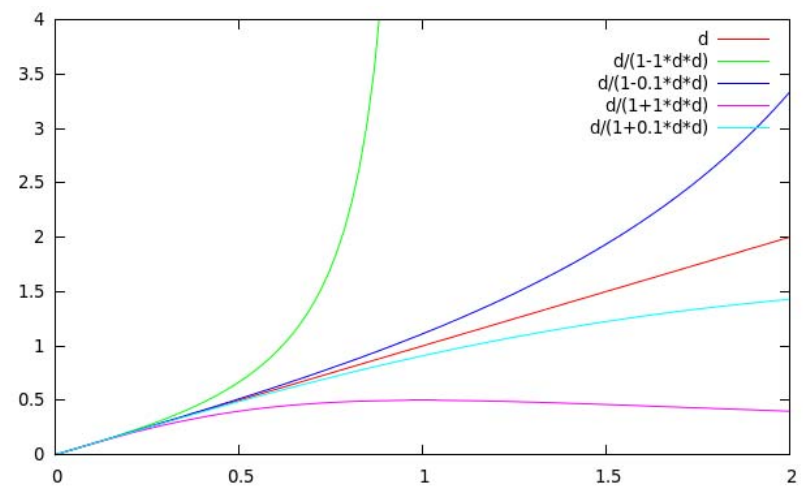

Figure 2. The undistorted distance to the $\mathrm{CoD}$ is plotted as a function of the distorted distance $d$ for different $\lambda$ according to the division distortion model (Eq.(2)). Please observe that for positive $\lambda$ (lower two curves) the undistorted radius is limited (the curves have a maximum). This leads to interesting consequences: Although every point can be undistorted according to this model, only those close to the distortion center can be distorted. The model implies a horizon beyond which undistorted points cannot be distorted (their distorted coordinates become complex). This can for instance happen when one wants to compute the distorted epipole, when the undistorted epipole is outside the horizon. Second, one can observe that in case of positive $\lambda$ each undistorted point has two distorted solutions to which it is compatible. However, since non-monotonic distortion curves do not make much sense, the one closer to the distortion center is the interesting one, since this is on the (useful) monotonic part of the curve and the other one can be considered an artifact by the model.

represents the squared distance to the radial distortion center, this center has to be known when working with these models. All of these approaches can cover the same family of radial distortion functions and thus the same family of lenses. The function of undistorted radius versus distorted radius is plotted for different values of $\lambda$ in Fig. 2.

The rational function undistortion model An extended family of functions has been introduced by Claus and Fitzgibbon $[8,7]$

$$
p_{u}=\left(\begin{array}{c}
x_{u} \\
y_{u} \\
1
\end{array}\right) \cong A\left(\begin{array}{c}
x_{d}^{2} \\
x_{d} y_{d} \\
y_{d}^{2} \\
x_{d} \\
y_{d} \\
1
\end{array}\right),
$$

$A$ is a $3 \times 6$ matrix of distortion coefficients (Eq.(3) in [8]):

$p_{u}=\left(\begin{array}{l}A_{11} x_{d}^{2}+A_{12} x_{d} y_{d}+A_{13} y_{d}^{2}+A_{14} x_{d}+A_{15} y_{d}+A_{16} \\ A_{21} x_{d}^{2}+A_{22} x_{d} y_{d}+A_{23} y_{d}^{2}+A_{24} x_{d}+A_{25} y_{d}+A_{26} \\ A_{31} x_{d}^{2}+A_{32} x_{d} y_{d}+A_{33} y_{d}^{2}+A_{34} x_{d}+A_{35} y_{d}+A_{36}\end{array}\right)$

Although not explicitly mentioned in [7, 8], that new model is a generalization of the division model and includes it by 
setting several coefficients to zero. This can be seen easily when we choose

$$
A_{\mathrm{d}}=\left(\begin{array}{cccccc}
0 & 0 & 0 & 1 & 0 & 0 \\
0 & 0 & 0 & 0 & 1 & 0 \\
\lambda & 0 & \lambda & 0 & 0 & 1
\end{array}\right) .
$$

Having 17 degrees of freedom (as the matrix $\mathrm{A}$ is defined only up to scale), the rational function model can approximate a much broader range of distortions. Having different coefficients for the $x^{2}$ and $y^{2}$ terms also means that is no longer a radially symmetric distortion model. While being much more powerful, the downside is the high number of parameters to estimate in calibration approaches and Claus and Fitzgibbon propose to start from a good prediction and do a gradient descent (however, in appendix A we propose a different method that does not depend on nonlinear optimization). On top, the parameters can be recovered only up to a homography and in the end, although epipolar lines are straightened, there is significant distortion left in their example images.

Division undistortion with unknown center In [3] it has been shown that the lifting and the radial fundamental matrix can be reformulated such that one can work with $\left(r^{\prime}\right)^{2}$, the squared distance to the origin of the image and thus need not know the radial CoD in advance. Rather, it is absorbed in the lifting matrix, or ultimately, in the radial fundamental matrix. In the Claus and Fitzgibbon framework this would mean that the second column of $A$ is zero (no mixed terms), the first and third column are equal and that all entries depend only on the distortion center $\left(d_{x}, d_{y}\right)$ and $\lambda$. More in detail, adapting $L$ from equation (9) of [3] we obtain

$$
\begin{gathered}
A_{\mathrm{c}}=\left(\begin{array}{cccc}
1 & & d_{x} & \lambda d_{x} \\
& 1 & d_{y} & \lambda d_{y} \\
& & 1 & \lambda
\end{array}\right)\left(\begin{array}{cccccc}
0 & 0 & 0 & 1 & 1 & -d_{x} \\
0 & 0 & 0 & -d_{y} \\
0 & 0 & 0 & & 1 \\
1 & 0 & 1 & -2 d_{x} & -2 d_{y} & d_{x}^{2}+d_{y}^{2}
\end{array}\right)= \\
\left(\begin{array}{cccccc}
\lambda d_{x} & 0 & \lambda d_{x} & 1-2 \lambda d_{x}^{2} & -2 \lambda d_{x} d_{y} & 1+\lambda d_{x}\left(d_{x}^{2}+d_{y}^{2}\right) \\
\lambda d_{y} & 0 & \lambda d_{y} & -2 \lambda d_{x} d_{y} & 1-2 \lambda d_{y}^{2} & 1+\lambda d_{y}\left(d_{x}^{2}+d_{y}^{2}\right) \\
\lambda & 0 & \lambda & -2 \lambda d_{x} & 2 \lambda d_{y} & 1+\lambda\left(d_{x}^{2}+d_{y}^{2}\right)
\end{array}\right) .
\end{gathered}
$$

Consequently, this model is in between the original division model and the rational function model. Since $A$ has only three degrees of freedom and the lifted space is thus only 4-dimensional (rather than 6-dimensional) we will stick to this model for the rest of the paper. As will be clear later, the radial property will allow us to obtain the complete parameters, in contrast to the rational function model. Properties of the model are visualized in Fig. 3.

\section{CoD From Straight Epipolar Lines}

We now consider a pair of images $\mathrm{A}$ and $\mathrm{B}$, at least one of them (say image A) having radial distortion (but not necessarily being described parametrically by any of the above

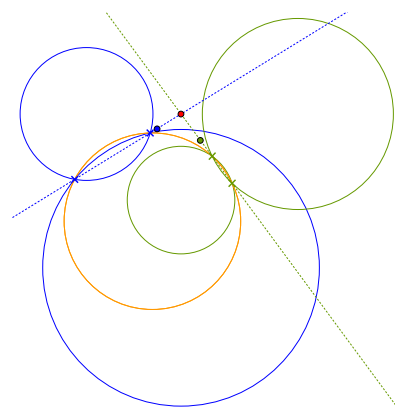

$\lambda>0$

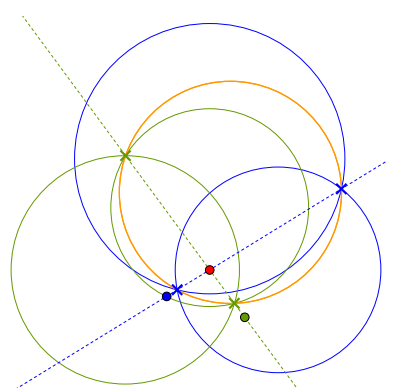

$\lambda<0$
Figure 3. Visualization of the radial distortion due to the division distortion model: The $\mathrm{CoD}$ is visualized in red. The distorted points are given by the intersection of two circles (green: circles for the epipoles; blue: circles for an arbitrary point). The distorted epipolar line becomes an epipolar circle (orange). Note that all four points (the two distorted epipoles and the two distorted points) lie on this circle.

mentioned models). Let us now project the line of sight in space for a point in image B into this distorted image and we obtain an epipolar curve in A. When we knew the distortion function we could undistort image A to image A' and in the latter image, all epipolar curves would be straight lines.

Recall that always all epipolar curves go through the epipole and now we look at the one that also includes the distortion center. Distortion happens in radial direction from the center to the epipole and thus within the line, but does not change the line as a whole. In the next section we are going to obtain a parametric description of the set of all epipolar curves, in which we can search for straight lines.

\subsection{Radial Fundamental Matrix}

We now choose the distortion model of Brito et al. and compute the radial fundamental matrix [3] between our image $\mathrm{A}$ and some other image (depending on the other image this could be the single-sided or two-sided radial fundamental matrix). For image correspondences $(p ; q)$ between image $\mathrm{A}$ and image $\mathrm{B}$ we obtain

$$
q^{T} F p=0,
$$

where $p$ is a lifted, 4D vector with distorted coordinates $\left(x_{d}, y_{d}, 1, x_{d}^{2}+y_{d}^{2}\right)^{T}$. For any point $q$ in the other image (besides the epipole)

$$
l_{A}=q^{T} F
$$

defines an epipolar curve in image $A$. More precisely, in this model, this is a circle ${ }^{2}$, because of the special structure of $F$. However, Eq. (8) is just a linear combination of the rows of $F$, weighted by the entries of $q$. Since $F$, as a radial fundamental matrix, has a two dimensional rowspace, we can

\footnotetext{
${ }^{2}$ Including degenerate circles with infinite radius (line), zero radius (point) or negative radius (no real point at all).
} 
easily extract two basis vectors $\left\{r_{1}, r_{2}\right\}$ for this rowspace and we can represent

$$
l_{A}=\alpha_{1} r_{1}+\alpha_{2} r_{2} .
$$

We can set $\alpha_{1}$ to 1 since the problem is defined only upto-scale. $l_{A}$ is a straight line only if it does not depend on quadratic entries, so its fourth entry must vanish. This provides a linear equation with a unique solution unless both last entries of $r_{1}$ and $r_{2}$ are zero. In this case, since $r_{1}$ and $r_{2}$ form a basis of the rowspace, the last column of $F$ must be entirely zero. We will come back to this case in the next section. In all other cases we obtain a unique straight line in the set of all possible epipolar curves. As argued before the distortion center must be on that line.

\subsubsection{Degenerate Cases}

In case image $A$ does not have distortion, then $\lambda$ is zero and consequently the last column of the radial fundamental will be zero. In that case all epipolar curves are straight and the distortion center is not defined. All epipolar curves are also straight if the epipole coincides with the distortion center.

Interestingly, these two cases cannot be distinguished, and this is again a property that is independent of the particular radial distortion function: under undistorted epipolar geometry each position on the epipolar line corresponds to a valid 3D point, so if we move a point away from the epipole this could mean it is just further away from the camera. Or, this could be by radial distortion. This typically happens with forward motion. This case of an entirely zero column or row can however easily be detected from the radial fundamental matrix.

\subsection{CoD from three (different) images}

As argued before, from a pair of images $A$ and $B$, we can constrain the position of the distortion center to a line in the image plane. To obtain the full coordinates of the distortion center we can intersect the line with another line. For instance, if we have another image $C$, the epipole in image $A$ will in general be different. Consequently, the straight epipolar curve will be different and by intersecting the two straight epipolar curves we obtain the distortion center. None of the three images used has to be calibrated, but we must be able to compute the radial fundamental matrix. For practical reasons, the lines should intersect ideally at a right angle, so the epipoles should be at different directions when viewed from the distortion center. This is the case, e.g. for A-to-B horizontal camera motion (epipole at $\mathrm{X}$-infinity) and A-to-C vertical camera motion (epipole at y-infinity). In contrast, all three camera centers being collinear is typically a (close to) degenerate situation, where the epipolar lines intersect at a small angle or fall entirely together. The principle is visualized in Fig. 1(left).

\subsection{CoD from two images of the same camera}

In case we have a video or multiple images taken by the same camera we can even extract the distortion center from a pair of two images. In each of the images we obtain the constraint that the distortion center must be on the straight epipolar line. However, in case we know that it is the same distortion center in both images, we can just intersect these two lines. Again, the lines should meet ideally at a right angle which is for instance the case for a pair of landscape and portrait pictures where the camera was moved in horizontal direction. The principle is again visualized in Fig. 1(right).

Note that this is a similar setting as studied by Fitzgibbon [10], however we also estimate the distortion center. Once the center is known, one could plug everything into the system proposed in [10] or, alternatively we show an easier way of obtaining $\lambda$ given the epipoles.

\subsection{Other Settings}

Homography between distorted and undistorted image In case the observed, unknown scene is planar, then two images are related by a homography. If only one of them contains radial distortion, the relation is a single-sided $3 \times 4$ radial homography [3]. This single-sided homography is relevant in sequential calibration algorithms, where one can "chain" new images to calibrated ones. We briefly sketch how the same algebraic construction can be exploited to obtain the $\mathrm{CoD}$. As before, straight lines in the image without distortion are mapped to (circular) curves in the distorted view. Again, all possible curves are actually defined by the row space of $H$, which is now three-dimensional. When looking for those curves that are straight lines, we obtain a 1D family of lines that all intersect at the distortion center. In projective space this $1 \mathrm{D}$ family of lines spans a 2D subspace, and since the distortion center must lie on all of the lines, it is the orthogonal complement of the 2D subspace.

More Images The methods presented here are meant as a proof of concept and show minimum requirements. If more images are available one could then of course intersect more than two lines or find the closest point to all of these lines. Practically, one would finally optimize over all parameters in a bundle adjustment, however this is out of the scope of this article and we focus on finding the initial values.

\subsection{Obtaining $\lambda$}

Once the $\mathrm{CoD}$ is known, we could in principle reformulate the whole problem and utilize one of the approaches for known CoD. However, given that we know the $\mathrm{CoD}$ and the two epipoles ${ }^{3}$, then, in the distorted image we are looking

\footnotetext{
${ }^{3}$ Because of the quadratic components there are in general two epipoles in the distorted image. They can be extracted from the right null space of $\mathrm{F}$, as explained in Sec. 4.2 of [3].
} 
for a lambda and an undistorted epipole consistent with the model. Since distortion happens in radial direction, we can compute the distances $d_{1}, d_{2}$ of both distorted epipoles to the distortion center and study the distortion as a 1D problem, where $u$ is the undistorted distance:

$$
u=\frac{d_{1}}{1+\lambda d_{1}^{2}}=\frac{d_{2}}{1+\lambda d_{2}^{2}} .
$$

Here $u$ is the distance of the undistorted epipole to the distortion center. solving for $\lambda$ yields

$$
\lambda=\frac{d_{2}-d_{1}}{\left(d_{1} d_{2}^{2}-d_{2} d_{1}^{2}\right)} .
$$

\subsection{Higher order distortion parameters}

As long as we have a truly radially symmetric distortion model, we can always play the same trick with the intersection of the straight epipolar curve to determine the distortion center. Therefore the above mentioned algorithms for extracting the distortion center remain valid even for higher order radial distortion models and one can also think of a higher order radial fundamental.

\section{Experiments}

In this section we present results from a proof-of-concept implementation of the given approach. For all of the experiments we estimate the (two-sided) radial fundamental matrix using direct linear transformation on normalized feature coordinates (similar to [12]) using all inliers.

Synthetic Data First, we verify whether it is actually possible to estimate the distortion center with synthetic data (see Fig. 4). The approaches for two and three images do not differ conceptually for the evaluation, so we present only results for the case of same distortion. As can be seen, in an ideal setting with no noise we can always extract the distortion center, which proves our general idea of finding straight epipolar curves. As we start to increase the noise of the feature correspondences however, we observe an instability meaning that at more than one pixel noise the given implementation produces very large errors, even when the radial fundamental is non-linearly optimized. In principle the displacement of correspondences is due to relative camera rotation and a) effects from baseline (disparity), b) effects from distortion, c) effects from noise. It is clear that in case the effects from noise come close to one of the other phenomena then the radial fundamental estimate (and thus the distortion center estimation) will be highly fragile.

\subsection{Constant Distortion / Video}

In this experiment we validate the approach with real data of a GoPro-Hero video camera with $1920 \times 1080$ pixels
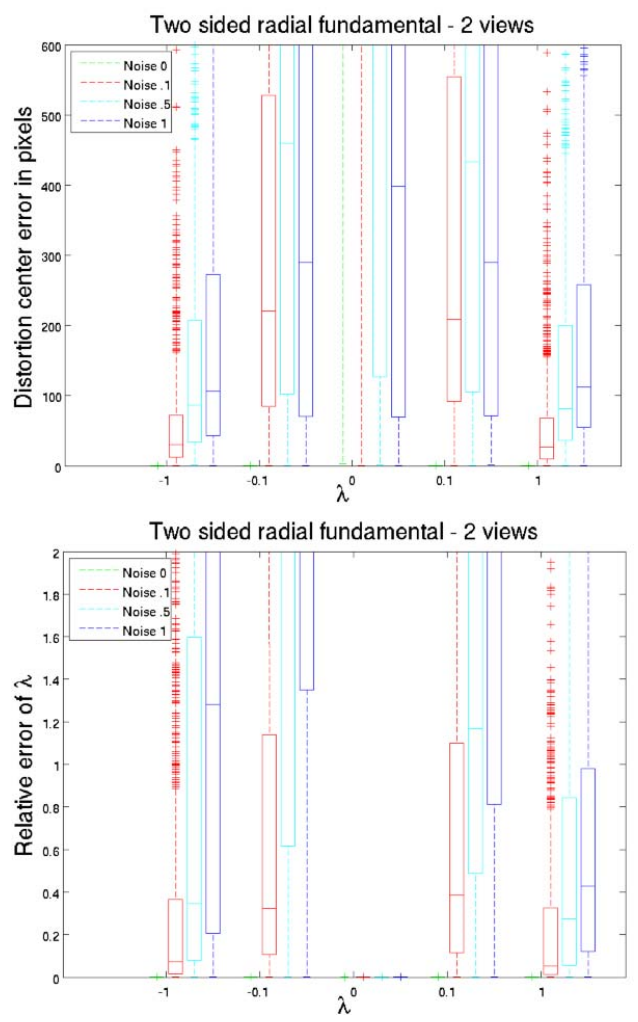

Figure 4. Synthetic experiments for stereo pairs of the same camera with moderate distortion $(|\lambda|=0.1)$ and strong distortion $(|\lambda|=1.0)$. We project a set of 3D points into two cameras (1000 randomized poses) and add Gaussian noise of the specified standard deviation to these coordinates. Then the two-sided radial fundamental is estimated from 100 correspondences and the distortion center is extracted. Top: Deviation of the distortion center. Bottom: Deviation of $\lambda$. The green plots for zero noise show zero error and are thus hardly visible.

resolution and very wide angle lens showing strong distortion. First we calibrate the distortion center using a chessboard by the method from Hartley and Kang [13]. We then take a video with the same camera of an office scene with several books and boxes. In this video we move the camera horizontally while rotating it around the optical axis ${ }^{4}$. We then track on the order of thousand local features through the video and compute radial fundamental matrices between frames from the beginning to an image 300 frames later in the sequence. From each pair we extract the distortion center and distortion coefficient as plotted in Fig. 5. Although we can see that the distortion centers cluster in a believable position, there is still a substantial offset to the position reported by the calibration-target-based method of Hartley and Kang. Besides the issues already observed in the synthetic data, here another reason for the deviation might be

\footnotetext{
${ }^{4}$ The rotation ensures epipolar lines will be horizontal in the beginning of the sequence and more diagonal later, allowing for intersecting them.
} 

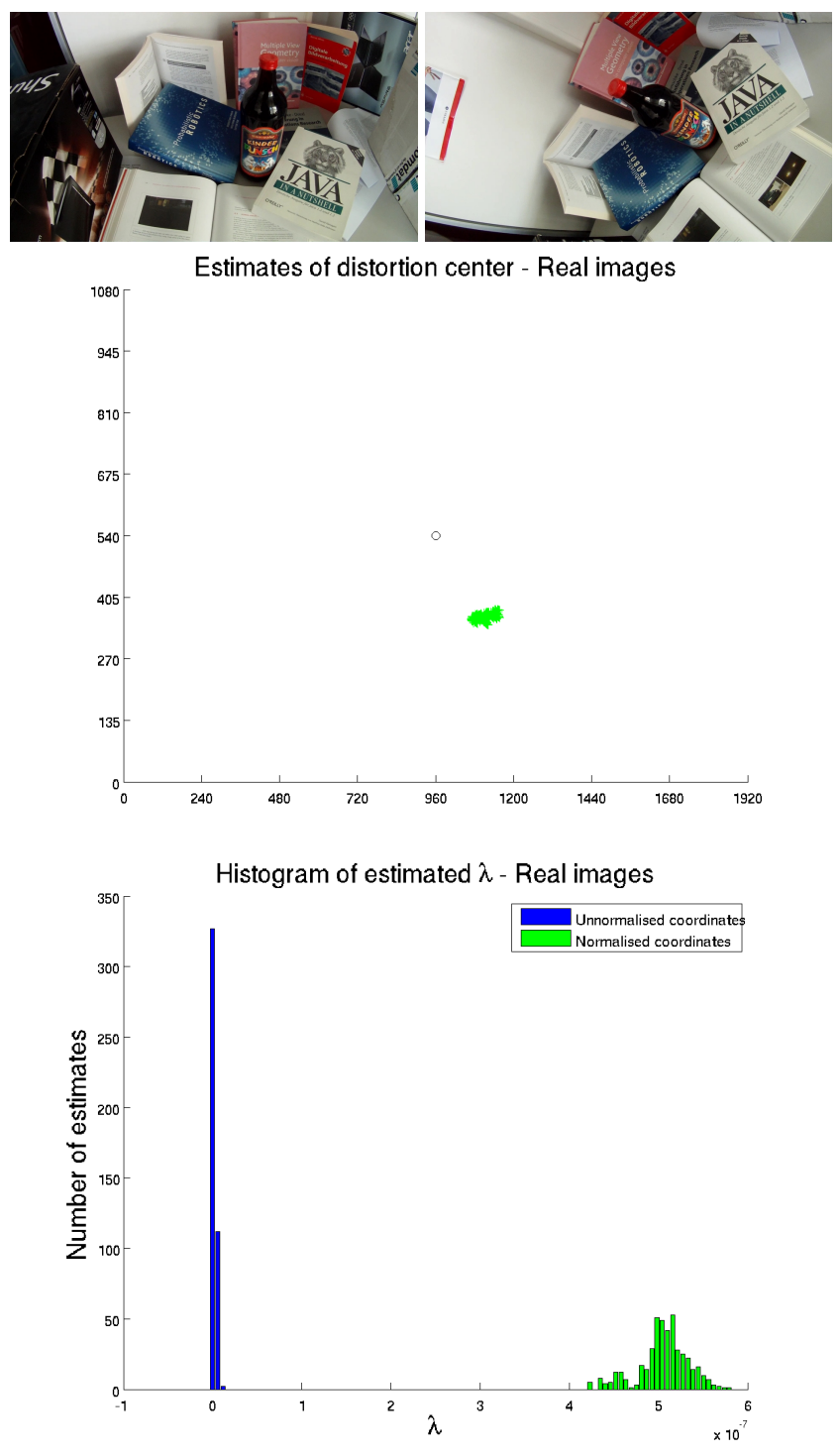

Figure 5. Video captured with a camera with strong distortion. While moving horizontally, the camera has been rolled around its optical axis. We compute the radial distortion center from pairs of views, where one is from the start of the sequence and the other from the end of the sequence. The centers cluster around (1108;360) close to the center of the image (also visualized) while we obtained a distortion center using the chessboard-based method of [13] at $(983 ; 530)$. We do not show the distortion centers when estimated without normalization here, because they are not useful and often very far away from the image, however we do plot the estimated $\lambda$ showing that normalization is important. Coordinates were normalized to $[-1 ; 1]$.

that the camera's actual distortion curve does not fit to the division distortion model.

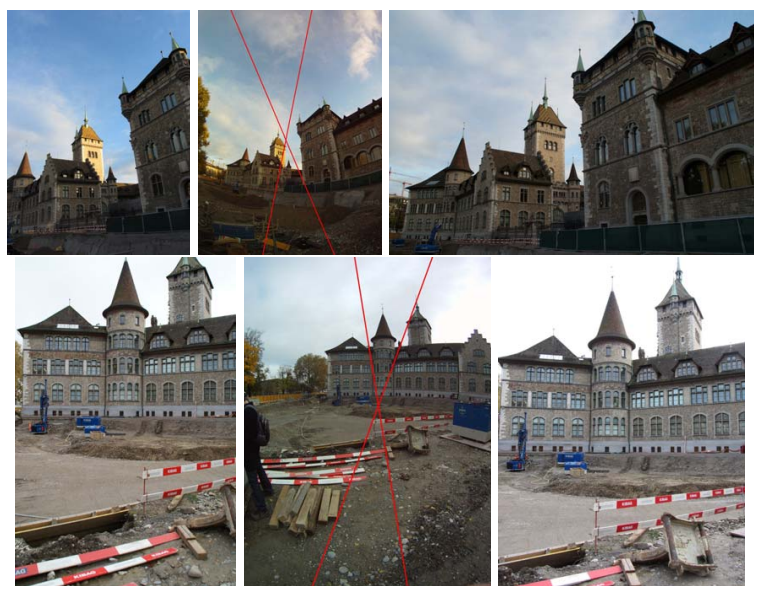

Figure 6. Two rows of three images taken with different cameras (with different amounts of radial distortion). For the center image the estimated radial distortion center is visualized by showing the two straight epipolar lines. As compared to a chessboard-based calibration by the method of [13] the distortion center is $2.5 \%$ away (top example) and $6.5 \%$ away (bottom example) from the ground truth position (fraction given with respect to image size).

\subsection{Different Distortions}

We also present results how to use the approach for three different cameras. Here we use two image triplets taken in a small baseline setting (with three different physical cameras). We then compute two radial fundamental matrices to the center camera and obtain a distortion center that is $2.5 \%$ resp. $6.5 \%$ away from the true distortion center (see Fig. 6).

\section{Conclusion}

Based on the observation that straight lines through the distortion center are fixed lines under any radial distortion model, we have derived constraints on the radial distortion center from epipolar geometry. Essentially, by intersecting two lines that must include the distortion center, its coordinates are revealed. In practice however, in particular with little distortion, circles with huge radius are difficult to distinguish from straight lines, so the procedure makes most sense in settings with high distortion. For high distortion, it is important to choose the appropriate distortion model, maybe with more than one parameter or directly aim at a parameter-free representation as in [13] (which then comes at the cost of requiring more views and many more correspondences). Consequently, while we could conceptually show that the approach can reveal the center it is by far not as reliable as chessboard-based methods [13], and future work should improve on the sensitivity and investigate on ways to robustify the algorithms to better cope with all kinds of noise (or systematic errors like from a slightly incorrect distortion model). 


\section{Acknowledgment}

Roland Angst is a recipient of the Google Europe Fellowship in Computer Vision, and this research is supported in part by this Google Fellowship.

\section{References}

[1] J. P. Barreto. A unifying geometric representation for central projection systems. Computer Vision and Image Understanding, 103(3):208-217, 2006.

[2] J. P. Barreto and K. Daniilidis. Fundamental matrix for cameras with radial distortion. In International Conference on Computer Vision, 2005.

[3] J. H. Brito, R. Angst, K. Köser, C. Zach, P. Branco, M. J. Ferreira, and M. Pollefeys. Unknown radial distortion centers in multiple view geometry problems. In Proceedings of the Asian Conference on Computer Vision, 2012.

[4] D. C. Brown. Close-range camera calibration. Photogrammetric Engineering, 37(8):855-866, 1971.

[5] T. A. Clarke and J. G. Fryer. The development of camera calibration methods and models. The Photogrammetric Record, 16(91):51-66, 1998.

[6] T. A. Clarke, X. Wang, and J. G. Fryer. The principal point and ccd cameras. The Photogrammetric Record, 16(92):293312, 1998.

[7] D. Claus and A. W. Fitzgibbon. A plumbline constraint for the rational function lens distortion model. In Proceedings of the British Machine Vision Conference, 2005.

[8] D. Claus and A. W. Fitzgibbon. A rational function lens distortion model for general cameras. In IEEE Conf. Computer Vision and Pattern Recognition, 2005.

[9] F. Devernay and O. Faugeras. Straight lines have to be straight. Machine Vision and Applications, 13(1):14-24, 2001.

[10] A. Fitzgibbon. Simultaneous linear estimation of multiple view geometry and lens distortion. In IEEE Conf. Computer Vision and Pattern Recognition, 2001.

[11] C. Geyer and K. Daniilidis. Structure and motion from uncalibrated catadioptric views. In IEEE Conf. Computer Vision and Pattern Recognition, 2001.

[12] R. Hartley. In defense of the eight-point algorithm. Pattern Analysis and Machine Intelligence, 19(6):580-593, 1997.

[13] R. Hartley and S. B. Kang. Parameter-free radial distortion correction with center of distortion estimation. Pattern Analysis and Machine Intelligence, 29(8):1309 -1321, 2007.

[14] R. I. Hartley and T. Saxena. The cubic rational polynomial camera model. In In Image Understanding Workshop, 1997.

[15] R. I. Hartley and A. Zisserman. Multiple View Geometry in Computer Vision. Cambridge University Press, 2000.

[16] J. Heikkila and O. Silven. A four-step camera calibration procedure with implicit image correction. In IEEE Conf. Computer Vision and Pattern Recognition, 1997.

[17] Z. Kukelova, M. Byröd, K. Josephson, T. Pajdla, and K. Aström. Fast and robust numerical solutions to minimal problems for cameras with radial distortion. Computer Vision and Image Understanding, 114(2):234 - 244, 2010.
[18] H. Li and R. Hartley. A non-iterative method for correcting lens distortion from nine-point correspondences. In Proceedings of OmniVision, 2005.

[19] B. Micusik and T. Pajdla. Estimation of omnidirectional camera model from epipolar geometry. In IEEE Conf. Computer Vision and Pattern Recognition, 2003.

[20] M. Pollefeys, R. Koch, and L. Van Gool. Self-calibration and metric reconstruction in spite of varying and unknown internal camera parameters. In Proceedings of the International Conference on Computer Vision, 1998.

[21] N. Snavely, S. M. Seitz, and R. Szeliski. Modeling the world from internet photo collections. International Journal of Computer Vision, 80(2):189-210, 2008.

[22] J.-P. Tardif, P. Sturm, M. Trudeau, and S. Roy. Calibration of cameras with radially symmetric distortion. Pattern Analysis and Machine Intelligence, 31(9):1552-1566, 2009.

[23] S. Thirthala and M. Pollefeys. Multi-view geometry of $1 \mathrm{~d}$ radial cameras and its application to omnidirectional camera calibration. In Proceedings of the International Conference on Computer Vision, 2005.

[24] R. Tsai. A versatile camera calibration technique for highaccuracy $3 \mathrm{~d}$ machine vision metrology using off-the-shelf $\mathrm{tv}$ cameras and lenses. Robotics and Automation, 3(4):323 344, 1987.

[25] Z. Zhang. Flexible camera calibration by viewing a plane from unknown orientations. In Proceedings of the International Conference on Computer Vision, 1999.

\section{A. Extraction of Lifting Matrix in Rational Undistortion Model}

Claus and Fitzgibbon [8] also consider the two-sided radial fundamental matrix arising from two images taken by the same camera, i.e. the distortion parameters are the same in both views. Their radial fundamental matrix is a 6 -by- 6 matrix, since the distorted points are lifted to $\mathbb{R}^{6}$. Specifically, according to Eq. (3), the two-sided radial fundamental looks like $\hat{\mathbf{F}}=\mathbf{A}^{T} \mathbf{F A} \in \mathbb{R}^{6 \times 6}$, where $\mathbf{F} \in \mathbb{R}^{3 \times 3}$ again denotes the standard fundamental matrix. While equation (23) in [8] suggests a non-linear optimization in order to extract the lifting matrix $\mathbf{A}$ from a given radial fundamental $\hat{\mathbf{F}}$, we argue that this can actually be done in closed-form. Let $\hat{\mathbf{F}} \in \mathbb{R}^{6 \times 6}$ be the two sided radial fundamental. Let $\mathbf{C} \in \mathbb{R}^{6 \times 2}$ and $\mathbf{R} \in \mathbb{R}^{6 \times 2}$ denote the two-dimensional column and row space of $\hat{\mathbf{F}}$, respectively. Then, since the lifting matrix $\mathbf{A}$ has a three-dimensional rowspace which is the underlying space for both the row- and column-space of $\hat{\mathbf{F}}$, we have $\operatorname{dim}(\operatorname{span}([\mathbf{C}, \mathbf{R}]))=3$. Hence, the three first left singular vectors $\mathbf{U}=\left[\mathbf{u}_{1}, \mathbf{u}_{2}, \mathbf{u}_{3}\right]$ of $[\mathbf{C}, \mathbf{R}]$ span the same space as the rows of $\mathbf{A}$, i.e. $\mathbf{A}=\mathbf{H U}^{T}$. In the setting used in [8], the radial distortion matrix $\mathbf{A}$ is only defined up to an arbitrary projective mapping of the image plane. Hence, the singular vectors in $\mathbf{U}^{T}$ represents a perfectly valid solution for the lifting coefficients in $\mathbf{A}$. 